\title{
Is Duration of Endotracheal Tube Intubation Using a Video Laryngoscope Different with a Direct Laryngoscope in Elective Surgery Patients During Pandemic Covid-19?
}

\author{
Zaky Hasan ${ }^{1}$, Zulkifli2*, Agustina Br Haloho², Irfannuddin $^{3}$ \\ ${ }^{1}$ Resident of Department of Anesthesiology and Intensive Therapy, Faculty of Medicine Universitas Sriwijaya, \\ Mohammad Hoesin Hospital, Palembang, Indonesia \\ ${ }^{2}$ Consultant of Department of Anesthesiology and Intensive Therapy, Faculty of Medicine Universitas Sriwijaya, \\ Mohammad Hoesin Hospital, Palembang, Indonesia \\ ${ }^{3}$ Department of Physiology, Faculty of Medicine Universitas Sriwijaya, Palembang, Indonesia
}

\author{
*Corresponding author: \\ Zulkifli \\ Consultant of Department of \\ Anesthesiology and Intensive \\ Therapy, Faculty of Medicine \\ Universitas Sriwijaya, Mohammad \\ Hoesin Hospital, Palembang, \\ Indonesia \\ Email: \\ $\underline{\text { dr_zulkifli_span@yahoo.co.id }}$
}

https://doi.org/10.37275/jacr.v1i2.142

\begin{abstract}
Introduction: Aerosol box is a usefull tools to prevent aerosol and droplet contaminations during laryngoscopy and intubation in COVID-19 pandemic. Video laryngoscope is recommended during this era to increase the operator and patient's distance during the procedure. However, many anesthesiologists still use direct laryngoscopes due to their availability and familiarity. This study aims to compare endotracheal tube intubation with video laryngoscope compared to direct laryngoscope in elective surgery patients.

Methods: This study was a quasi-experimental study. The sample size in each group (video and direct laryngoscope) was 35 subjects. The primary outcome of this study was to compare the duration of intubation between video laryngoscope and direct laryngoscope, while the secondary outcome was to evaluate complications such as broken teeth and sore throat 24 hours post intubation.

Results: Direct laryngoscope had shorter intubation duration than video laryngoscope [12.3 (8.9-21.0) vs $13.3(11.4-21.6 ; \mathrm{p}=0.07)$ respectively. Two complication reported on video laryngoscope groups.

Conclusion: Duration of ETT intubation using a direct laryngoscope was shorter during intubation with aerosol box compared to video laryngoscope in elective surgery patients. The box which was being used may limit the space of movement making it difficult to intubate.
\end{abstract}

Keyword: Aerosol Box, COVID-19, Direct Laryngoscope, Intubation, Video Laryngoscope 


\section{Introduction}

Endotracheal tube (ETT) intubation is an act of inserting a special tube into the trachea so that the airway is patent and the breath is easily controlled. ${ }^{1}$ Laryngoscopy and intubation are not risk-free procedures where both are high-risk procedures that result in morbidity and mortality. Endotracheal intubation often causes trauma to the upper airway mucosa, which manifests as postoperative symptoms. Some of the symptoms that patients complain of include: sore throat, cough, and hoarseness. Christensen, et al. and Loeser, et al. have reported an incidence of 21-65\% airway management failure for a rapid duration in the patient may lead to serious complications or death. ${ }^{1,2}$

Long duration and failure of intubation have the potential which leads to serious problems until death, therefore an alternative tool is needed that can minimize the incidence of failure and complications during the intubation procedure. Several methods can make it easier to visualize the larynx during the intubation procedure, including flexible fiberoptic bronchoscopy, stylet optic, and video laryngoscope (VL). ${ }^{3,4}$ Video laryngoscope is a method to improve glottis visualization and increase intubation success compared to direct laryngoscope (DL). The intubation method using VL is a laryngoscope method with a camera that is fixed and attached to the laryngoscope handle. Silverberg and Arulkumaran's study found that VL resulted in a higher first intubation success rate than DL. ${ }^{1,5-7}$

Although VL is proven to have a better field of view than DL, there is still controversy in its function, this is based on Lascarrou's 2017 Randomized Controlled Trial which found that VL did not produce a tracheal intubation success rate in the first trial that was higher than DL and associated with a higher complication rate. Therefore, it is controversial in the success of intubation in the first trial and in preventing complications in patients. ${ }^{1,5-7}$

In carrying out general anesthesia during the pandemic coronavirus disease (COVID), intubation, and laryngoscopy in COVID-19 patients requires fast and short action presents a challenge for anesthetists. The use of VL allows the operator to increase the distance to the patient's airway compared to DL and can help reduce the aerosol risks. Besides, compared to DL, VL can provide a better glottic view, can reduce intubation attempts in unexpected airway difficulties, and reduce laryngeal trauma, making this technique safer for the patients. The use of an aerosol box described by Lai Hsien usually consists of a transparent plastic cube that covers the patient's head and shoulders, with an access hole for an intubation procedure specialist's arm and sometimes an additional hole for an assistant can reduce the 


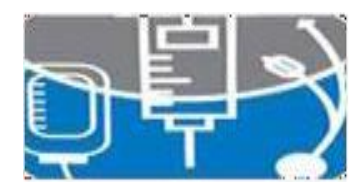

\section{Journal of Anesthesiology \& \\ Clinical Research}

risk of spreading COVID-19. ${ }^{8-10}$

Video laryngoscope is recommended during the COVID-19 pandemic era since it increases the distance between operator and patient during the procedure. However, many anesthesiologists still use direct laryngoscopes due to their availability and familiarity. Moreover, the use of aerosol boxes during laryngoscopy may limit operator space. This stıdy aims to compare endotracheal tube intubation with video laryngoscope compared to direct laryngoscope in elective surgery patients.

\section{Material and methods}

This study was a quasi-experimental study, the data taken was the primary data from the direct assessment of the patient who was subjected to the intubation procedure. This study was conducted in the central operating room of Mohammad Hoesin hospital during the period September - November 2020 until the number of samples was reached. The study population was all elective surgery patients under general anesthesia using intubation and met the inclusion criteria at Dr. Mohammad Hoesin Palembang with inclusion criteria, namely patients who will undergo elective surgery with general anesthesia intubation, aged between 18 to 65 years, have ASA I / II physical status, with Mallampati I / II crit eria, and Cormack Lehane $1 / 2$ criteria, and TMD $\geq 6 \mathrm{~cm}$. The exclusion criteria in this study were patients who refused to undergo general anesthesia with intubation using a video laryngoscope. The drop-out criteria in this study were patients with failed intubation (ETT into the stomach), patients with intraoperative complications (such as bleeding, shock, cardiac arrest), and patients who could not be extubated.

Sampling was carried out through block randomization, namely by dividing the groups of blockers consecutively where the researcher selects samples that meet the study criteria until the sample was reached. Before intubation, the study subjects were given a box pillow, the operating table was set at the same level as the distance between the xiphoid process and the operator's umbilicus, accompanied by an assistant to the right of the operator (helping to position and provide hood and ETT), induction was carried out by a resident with yellow level competence (semester 5-6) first gradually starting from pre- oxygenation for 3 minutes, then induction of anesthesia was started with the administration of $2 \mathrm{mcg} / \mathrm{KgBW}$ of fentanyl and $2 \mathrm{mg} / \mathrm{kg} \mathrm{BW}$ of propofol after the study subjects fell asleep, positive pressure ventilation was carried out using a face mask and $100 \%$ oxygen. If the study subject could be ventilated using a face mask, then administered atracurium $0.5 \mathrm{mg} / \mathrm{kg} \mathrm{BW}$, and the anesthetic was added with 


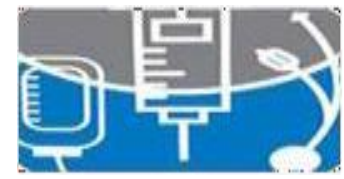

\section{Journal of Anesthesiology\& \\ Clinical Research}

volatile sevoflurane $2 \mathrm{Vol} \%$. After 3 minutes and the onset of drugs was achieved, direct laryngoscopy was performed without cricoid pressure using a video laryngoscope to see the visualization of the larynx performed by study participants, 7th and 8th-semester anesthesiologist resident physicians (green level). The data that has been collected will be analyzed using SPSS version 22.0.

\section{Results}

This study is a quasi-experimental study that aims to assess the comparison of the duration of ETT intubation using VL and DL during intubation in elective surgery patients at Mohammad Hoesin hospital where the researcher intervened with random control. In this study, a total of 70 samples were found that met the inclusion and exclusion criteria.

Table 1. Characteristics of study subjects

\begin{tabular}{lccc}
\hline \multirow{2}{*}{ Variable } & \multicolumn{2}{c}{ Laryngoscope } & \multirow{2}{*}{ p_Value } \\
\cline { 2 - 3 } & $\begin{array}{c}\text { Direct } \\
\text { Laryngoscope }\end{array}$ & $\begin{array}{c}\text { Video } \\
\text { Laryngoscope }\end{array}$ & \\
\hline Age & & & $0.383^{*}$ \\
$\quad$ Mean \pm SD & $48.25 \pm 13.05$ & $45.22 \pm 15.69$ & \\
Median & 52.00 & 42.00 & \\
Min-Max & $18.00-71.00$ & $20.00-81.00$ & \\
Gender & & & $0.467^{* *}$ \\
Female & $22(62.9 \%)$ & $19(54.3 \%)$ & \\
Male & $13(37.1 \%)$ & $16(45.7 \%)$ & $0.303^{* *}$ \\
ASA & & & \\
I & $22(62.9 \%)$ & $19(54.3 \%)$ & $0.673^{* * *}$ \\
II & $13(37.1 \%)$ & $16(45.7 \%)$ & \\
Mallampati & & & $1.000^{* * *}$ \\
I & $31(88.6 \%)$ & $33(94.3 \%)$ & \\
II & $4(11.4 \%)$ & $2(5.7 \%)$ & $0.382^{* *}$ \\
TMD & & & \\
$\geq 6 \mathrm{~cm}$ & $34(97.1 \%)$ & $34(97.1 \%)$ & $1(2.9 \%)$ \\
$<6 \mathrm{~cm}$ & $1(2.9 \%)$ & & \\
Cormack Lehane & & & \\
\hline
\end{tabular}




\begin{tabular}{l} 
Journal of Anesthesiology \& \\
Clinical Research \\
\hline I \\
II
\end{tabular}

Note: * Independent t-test; **: Chi-Square; ***: Fisher's Exact

This study found that the mean age in the DL group was 48.25 \pm 13.05 and in the VL group was $45.22 \pm 15.69$. A normality test was carried out and it was found that the age variable was normally distributed, then an independent t-test was carried out and it was found that there was no difference in age between the two groups.

In the gender distribution of the laryngoscopy technique, it was found that $62.9 \%$ of the subjects were female and $37.1 \%$ of the subjects were male in the DL group, while there were $54.3 \%$ female subjects and 45.7\% subjects. male in the VL group. In the Chi-Square analysis test, it was found that there was no relationship between gender and the laryngoscope group.

In the distribution of ASA to the laryngoscopy technique, it was found that $62.9 \%$ of subjects with ASA I and $37.1 \%$ of subjects with ASA II were in the DL group, whereas there were $74.3 \%$ subjects with ASA I and $25.7 \%$ subjects with ASA II. in the VL group. In the Chi-Square analysis test, it was found that there was no relationship between ASA and the laryngoscope group.

In the Mallampati distribution of the laryngoscopy technique, it was found that as many as $88.6 \%$ of subjects with Mallampati I and 11.4\% of subjects with Mallampati II were in the DL group, whereas there were $94.3 \%$ subjects with Mallampati I and 5.7\% subjects with Mallampati II. in the VL group. In Fisher's Exact analysis, it was found that there was no relationship between Mallampati and the laryngoscope group.

In the distribution of TMD to the laryngoscopy technique, it was found that as many as $97.1 \%$ of subjects with TMD $>6 \mathrm{~cm}$ and $2.9 \%$ of subjects with TMD $<6 \mathrm{~cm}$ in the DL group, whereas there were $97.1 \%$ of subjects with TMD $>6 \mathrm{~cm}$ and 2, 9\% of subjects with TMD $<6 \mathrm{~cm}$ in the VL group. In Fisher's Exact analysis, it was found that there was no relationship between TMD and the laryngoscope group.

In the distribution of CormackLehane to the laryngoscopy technique, it was found that $74.3 \%$ of subjects with CormackLehane I and $25.7 \%$ of subjects with CormackLehane II were in the DL group, whereas there were $82.9 \%$ of subjects with CormackLehane I and $17.1 \%$ of subjects with CormackLehane in the DL group. VL group. In the Chi-Square analysis test, it was found that there was no relationship between CormackLehane and the laryngoscope group. 


\section{Distribution of the duration of intubation to the laryngoscopy technique}

Table 2. Distribution of the duration of intubation to the laryngoscopy technique

\begin{tabular}{cccc}
\hline \multirow{2}{*}{$\begin{array}{c}\text { Duration of } \\
\text { intubation (seconds) }\end{array}$} & \multicolumn{2}{c}{ Laryngoscope } & \multirow{2}{*}{ p_Value } \\
\cline { 2 - 3 } & Direct laryngoscope & Video laryngoscope & \\
\hline Mean \pm SD & $12.87 \pm 2,93$ & $14.09 \pm 2.54$ & 0.007 \\
Median & 12.30 & 13.30 & \\
Min - Max & $8.90-21.00$ & $11.40-21.60$ & \\
\hline
\end{tabular}

Note: Analysis using Mann-Whitney, $\mathrm{p}<0.05$ = significant

The distribution of the intubation duration to the laryngoscopy technique can be seen in table 2 . It was found that the intubation duration was not normally distributed in each group with a value of $\mathrm{p}=$ 0.000 in the DL group and $\mathrm{p}=0.000$ in the VL group. The mean value in the DL group was 12.30 (8.90 21.00) and in the VL group was 13.30 (11.40 - 21.60). The Mann-Whitney test was performed and it was found that there was a significant difference in between two groups.

\section{Distribution of post-intubation complications to the laryngoscopy technique}

The distribution of intubation complications to the laryngoscopy technique can be seen in table 3 .

Table 3. Distribution of intubation complications to the laryngoscopy technique

\begin{tabular}{lccc}
\hline \multirow{2}{*}{ Intubation complications } & \multicolumn{2}{c}{ Laryngoscope } & p_Value \\
\cline { 2 - 3 } & $33(94.3 \%)$ & $35(100 \%)$ & 0.151 \\
No complications & $2(5.7 \%)$ & $0(0 \%)$ & \\
Sore throat & Direct laryngoscope & Video laryngoscope & \\
\hline
\end{tabular}

Note: Analysis using Fisher's Exact, $\mathrm{p}<0.05$ = significant

In the distribution of swallowing pain to the laryngoscopy technique, it was found that $94.3 \%$ of subjects without swallowing pain and $5.7 \%$ of subjects with swallowing pain were in the DL group, whereas there were as many as $100 \%$ of subjects without swallowing pain in the VL group. In the Chi-Square analysis test, it was found that there was no significant relationship between intubation 


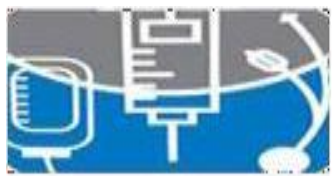

\section{Journal of Anesthesiology\& \\ Clinical Research}

complications and the laryngoscope.

\section{Discussion}

In the distribution of the characteristics of the study subjects on the laryngoscopy technique, there were no significant variables that had a relationship or difference between the direct laryngoscope groups and the video laryngoscope groups. This was because the sampling technique used in this study was random, so it did not target certain groups (direct laryngoscope or video laryngoscope). In the distribution of the intubation duration of the study subjects to the laryngoscopy technique, it was found that the intubation duration was not normally distributed in each group with a value of $p=0.000$ in the DL group and $\mathrm{p}=0.000$ in the VL group. The mean score in the DL group was 12.3 (8.9 - 21.0) and in the VL group was 13.3 (11.4 - 21.6). The Mann-Whitney test was performed and it was found that there was a mean difference between the two groups with a value of $p=0.007$.

This result is different from Janz's study in 2016 which found that VL did not reduce intubation time (126 seconds, IQR 89 - 197) compared to DL (153 seconds, IQR 93-253, p = 0.13) as a whole (several intubation attempts) or in patientsrequiring only one trial (105 seconds, IQR 75 - 150 versus 112 seconds, IQR $86-156, \mathrm{p}=0.45) .^{11}$

Several other studies such as the Cochrane review found that VL compared to DL is known to shorten the duration of intubation as well as reduce intubation failure, reduce the likelihood of laryngeal/airway trauma, increase easy visualization of the larynx, the duration of intubation required in VL is shorter at 101.1 seconds. versus DL 126.8 seconds. ${ }^{12-15}$ Wajdi's study described a statistically significant length of intubation with an average VL of 32 seconds with a DL of 52 seconds where (p $<0.001) .{ }^{16}$

Jafra's study in 2018 found that successful intubation durations (seconds) were $24.89 \pm 5.574$ in the VL Glidescope group and $20.68 \pm 3.637$ in the DL group $(\mathrm{P}<0.001)$ found a statistically significant difference. Jafra also found a significant improvement in glottic appearance with the GlideScope (as assessed by POGO scores of 66.71 \pm 29.929 and $94.40 \pm 10.476$ in the VL group and $75.85 \pm 26.969$ and $74.20 \pm 29.514$ in the DL group and Cormack-assessment. Lehane $[\mathrm{P}<0.001])$. Based on that scores, VL Glidescope had easier intubation. Jafra also concluded that the VL GlideScope offers advantages over DL 


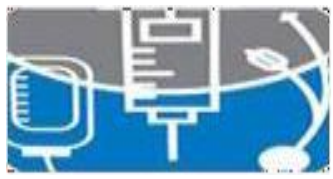

\section{Journal of Anesthesiology \&}

in terms of the view of the larynx and the difficulty encountered at intubation. ${ }^{37}$

Misirlioglu's study in 2016 also found the same thing, that the duration of intubation using the VL Glidescope was longer than using DL Macintosh in patients who were going to undergo ear, nose, and throat surgery with a p-value of $<0.05 .{ }^{18}$ A similar study conducted by Dasthi in 2014 found that out of 59 patients, intubation time was longer in the VL Glidescope group $(9.80 \pm 1.27$ seconds $)$ than in the DL Macintosh group $(8.20 \pm 1.17$ seconds) $(\mathrm{P}<0.05)$ in patients undergoing elective surgery with hypertension. ${ }^{19}$

The difference in this study is due to obstacles in carrying out intubation during this pandemic. During this pandemic, intubation procedures and laryngoscopes require operators to use aerosol boxes to reduce aerosol exposure from COVID-19 itself. The use of this box hinders arm movement in using a video laryngoscope due to the size of the video laryngoscope which is generally quite large compared to direct laryngoscopes, so in this study, it was found that direct laryngoscopes were superior in intubation with an aerosol box. The operator in this study was also a resident at the green level or at the final level who could be categorized as someone skilled in performing intubation. This may make the duration of using a video laryngoscope not much different if it is not using an aerosol box when compared to a direct laryngoscope.

A study by Nakanishi in 2020 found no difference between intubation duration despite using an aerosol box of 30 [26-32] seconds for Macintosh, 29 [26-32] seconds for i-view, and 29 [25-31] seconds for C-MAC $(\mathrm{p}=0.247)$. The success rate was $95 \%-100 \%$ without significant difference $(\mathrm{p}=0.135)$. The i-view and CMAC video laryngoscopes show higher Cormack-Lehane scores and lower subjective disturbance scale scores than Macintosh laryngoscopes. ${ }^{20}$

Another opinion by Madabushi found aerosol box had longer intubation duration than no aerosol box patients [42 (CI 19.2 - 64.8) seconds vs 52.1 (CI 26.1 - 78) seconds]. This difference of 10.1 seconds, according to Madabushi, falls into an apneic period that can be tolerated by most of the patients. ${ }^{21}$ Another systematic review by Zheng Jie also found that aerosol box could prolong the intubation duration (MD = 4.0 seconds; $95 \%$ CI: $2.4-5.6 ; \mathrm{p}<0.001) .^{22}$

In this study, it was also found that as many as $94.3 \%$ of subjects without swallowing pain and $5.7 \%$ of subjects with swallowing pain were in the direct laryngoscope group, and there were as many as $100 \%$ of subjects without swallowing pain in the video laryngoscope group. In the Chi-Square analysis 


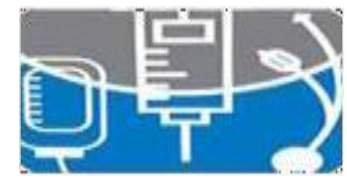

\section{Journal of Anesthesiology \& \\ Clinical Research}

test, it was found that there was no significant relationship between intubation complications and the laryngoscope. Panwar's study in 2020 found that the incidence of postoperative complications such as swallowing pain was less in the video laryngoscope group, namely 13 (26\%) were in grade 1 swallowing pain, compared to the direct laryngoscope group which was 32 (64\%) in swallowing pain. Grade 1 and 8 (16\%) patients had grade 2 swallowing pain. The analysis test found a significant difference. The curvature of the video laryngoscope blade and the presence of a camera causes less force, less tissue trauma and resulting in less painful swallowing. ${ }^{23}$

The study by Najafi in 2014 found that video glidescope laryngoscopes reduced the incidence of swallowing pain at 1 hour, 6 hours, 24 hours, and 48 hours post-surgery with the ratio of video laryngoscopes and direct laryngoscopes respectively, 29 (19.3\%) vs 42 (28\%) in the 1-hour group; 42 (28\%) vs 81 (54\%) in the $6 \mathrm{hr}$ group; $34(22.7 \%)$ vs 81 (54\%) in the 24 hours group and 28 (18.7\%) vs 49 $(32.7 \%)$ in the 48 hours postoperative group with $\mathrm{p}$ values of $0.08,<0.001,<0.001$, and 0.006 , respectively. ${ }^{24}$

\section{Conclusions}

In this study, it was found that the median duration of ETT intubation using a video laryngoscope was 13.3 (11.4 - 21.6) seconds, while the median duration of ETT intubation using a direct laryngoscope was 12.3 (8.9 - 21.0) seconds. There was difference in duration of intubation between the video and direct laryngoscope, the value of $\mathrm{p}=0.007$ ( $\mathrm{p}<0.05$ ) was statistically significant using the Mann-Whitney test. Comparison of post-intubation complications between video laryngoscopes and direct laryngoscopes, $\mathrm{p}=$ 0.151 ( $\mathrm{p}>0.05$ ) was not statistically significant using the Fisher Exact test.

\section{References}

1. Seong BM, Jin WH, Chae-Man L, Younsuck K, Sang-Bum H. Video laryngoscopy versus direct laryngoscopy for first-attempt tracheal intubation in the general ward. Annals of Intensive Care. 2018;8(1):1-11.

2. Simpson GD, Ross MJ, McKeown DW, Ray DC. Tracheal intubation in the critically ill: a multicenter national study of practice and complications. Br J Anaesth. 2012;108(5):792-9. 


\section{Journal of Anesthesiology \&}

3. Morgan GE, Mikhail MS, Murray MJ. Airway management. In: Stauss M, Lebowitz H, Boyle P, editors. Clinical Anesthesiology 6th ed. New York.: McGraw-Hill; 2018:315-29

4. Channa, AB. Video laryngoscopes. Saudi J Anaesth. 2011; 5(6):357.

5. Silverberg MJ, Li N, Acquah SO, Kory PD. Comparison of video laryngoscopy versus direct laryngoscopy during urgent endotracheal intubation: a randomized controlled tr ial. Crit Care Med. 2015;4(3):636-41.

6. Arulkumaran N, Lowe J, Ions R, Mendoza M, Bennett V, Dunser MW. Video laryngoscopy versus direct laryngoscopy for emergency orotracheal intubation outside the operating room: a systematic review and meta-analysis. Br J Anaesth. 2018;120(4):712-24.

7. Lascarrou JB, Boisrame-Helms J, Bailly A, Le Thuaut A, Kamel T, Mercier E, et al. Video laryngoscopy vs direct laryngoscopy on successful first-pass orotracheal intubation among ICU patients: a randomized clinical trial.JAMA. 2017;31(7):483-93.

8. Arif SK, Muchtar F. Buku pedoman penanganan pasien kritis Covid-19. PERDATIN. 2020;1(April):188.

9. Begley JL, Lavery KE, Nickson CP, Brewster DJ. The aerosol box for intubation in coronavirus disease 2019 patients: an in-situ simulation crossover study. Anaesthesia, 2020;75(8):1014-1021.

10. Hamal, Pawan Kumar, Rashan BC, Nabin P, Dipendra P, Gentle S S. An affordable video laryngoscope for use during the COVID-19 pandemic. Lancet Glob Health. 2020;8(7)893-4.

11. Janz DR, Semler MW, Lentz RJ. Randomized trial of video laryngoscopy for endotracheal intubation of critically ill adults. Crit Care Med. 2016; 44(11):1980-7.

12. Lewis S, Butler A, Parker J, Cook TM, Smith AF. Video laryngoscopy versus direct laryngoscopy for adult patients requiring tracheal intubation. Cochrane Database Syst Rev. 2016;11(6):81-93.

13. Lewis S, Butler A, Parker J, Cook TM, Smith AF. Video laryngoscopy versus direct laryngoscopy for adult patients requiring tracheal intubation: a Cochrane systematic review. Br J Anaesth. 2017; 119(8):369-83.

14. Nouruzi-Sedeh P, Schumann M, Groeben H. Laryngoscopy via Macintosh blade versus Glidescope: success rate and time for endotracheal intubation in untrained medical personnel. Anesthesiology 


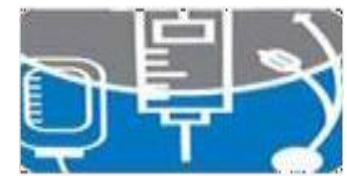

\section{Journal of Anesthesiology\& \\ Clinical Research}

2009;1(10):32-7.

15. Ferawati, Dedi F Y, Erwin P. Perbandingan metode pengajaran review video dengan tanpa review video terhadap keberhasilan dan lama intubasi pada mahasiswa program studi pendidikan dokter. J Anestesi Perioperatif. 2019; 7 (3): 147-152.

16. Wajdi F, Fitri D, Suwarman. Perbandingan intubasi endotrakea menggunakan clip-on smartphone camera videolaryngoscope dengan laringoskop macintosh pada manekin. J Anestesi Perioperatif. 2018;6(1):27-33.

17. Jafra, AS. A prospective randomized controlled study to evaluate and compare glidescope with Macintosh laryngoscope for ease of endotracheal intubation in adult patients undergoing elective surgery under general anesthesia. Saudi J Anaesth. 2018;12 (2):272

18. Misirlioglu G, Sen O. Comparison of glidescope video laryngoscopy and Macintosh laryngoscope in ear-nose and throat surgery. Ir J Med Sci. 2016;185(3):729-33.

19. Dashti M, Amini S, Azarfarin R, Totonchi Z, Hatami M. Hemodynamic changes following endotracheal intubation with GlideScope video-laryngoscope in patients with untreated hypertension. Res Cardiovasc Med. 2014;3(2):1-5.

20. Nakanishi, Toshiyuki, Yoshiki S, Yuji K, Kazuya S. Macintosh Laryngoscope, i-View ${ }^{\mathrm{TM}}$, and CMAC® Video Laryngoscopes for Tracheal Intubation with an Aerosol Box: A Randomized, Crossover, Manikin Study. Research Square2020:1-11.

21. Madabhushi, P., Kinthala, S., Ankam, A. et al. Time to adapt in the pandemic era: a prospective randomized non -inferiority study comparing time to intubate with and without the barrier box. BMC Anesthesiol 2020;20(232):1-8.

22. Lim, Zheng Jie, Mallikarjuna PR, Dharsi K, Kiran S, Ashwin S. Impact of an Aerosol Box on Time to Tracheal Intubation: Systematic Review and Meta-Analysis. British Journal of Anaesthesia. 2021;126(3).

23. Panwar N, Vanjare H, Kumari M, Bhatia V S, Arora KK. Comparison of video laryngoscopy and direct laryngoscopy during endotracheal intubation- a prospective comparative randomized study. Indian J of Clin Anaesth. 2020;7(3):438-43. 


\section{Journal of Anesthesiology \& \\ Clinical Research}

24. Najafi A, Imani F, Makarem J, Khajavi MR, Etezadi F, Shirin H, et al. Postoperative sore throat after laryngoscopy with macintosh or glide scope video laryngoscope blade in normal airway patients. Anesth Pain Med. 2014;4(1):1-4. 